\title{
EDITORIAL
}

\section{Proteomics: The Science of Functional and Structural Characterization of Proteins}

\author{
Mario Sergio Palma (iD $ه$ \\ Guest Editor of this special edition of BrJAC dedicated to Omics Tools \\ Full Professor of Proteomics \\ Department of Basic and Applied Biology, Institute of Biosciences of Rio Claro \\ São Paulo State University (UNESP), Rio Claro, SP, Brazil
}

The science of proteins was born many years ago as a branch of biochemistry, initially associated with medical applications. At that time, the standard protocols required the initial isolation and purification of an individual protein followed by the use of classical approaches of protein chemistry for amino acid sequencing and structural characterization. The analytical strategies were based on the use of large and low-performance chromatography columns and many spot tests for the chemical characterization of individual amino acids. This usually required very large amounts (grams/kilograms) of biological material in the initial steps of protein isolation and purification. At the time, most sequencing methods were based on the application of controlled chemical degradation protocols followed by thin layer chromatography of protein/ peptide hydrolysates. At first, all these analytical steps were operated manually; later, protein science adopted automation. However, the analytical strategies were focused on the individual investigation of each protein, which made progress on the structural characterization of each protein very slow (in general, 3-4 years of work were necessary to achieve the complete sequencing of a protein).

The initial application of mass spectrometry for peptide sequencing was made by using $\mathrm{N}$-terminaldirected acid hydrolysis of tryptic peptides (obtained by the proteolysis of a purified protein), followed by acid esterification to make them sufficiently volatile to be analyzed by GC-MS. Many important proteins were sequenced using this strategy.

Another aspect that must be emphasized was the success of DNA sequencing projects abroad that gave rise to several genome projects; these initiatives resulted in many different DNA databanks. The use of the universal genetic code to translate DNA sequences virtually into protein sequences, together with the development of algorithms for carrying out this translation and sequence alignment, contributed to create novel protein databanks. To give an idea about the impact of these initiatives, by the end of the 1990s the most-consulted protein databanks contained some hundreds of protein sequences; these new protein sequences generated by the virtual translation of deposited DNA sequences generated many thousands of protein sequences in a few years, achieving currently around 200 million entries. This progress represented an important boost for the popularization of protein science.

The immediate consequence was the combination of 2D electrophoresis of proteins (for the separation of large numbers of individual proteins) with protocols for in-gel digestion, followed by mass spectrometry analysis of the proteolytic peptides; this strategy resulted in the first experimental approach for the simultaneous identification of large numbers of proteins (peptide mass fingerprinting). At this time, the term "genomics" associated with studies on DNA sequencing started to be used, this meaning the complete DNA sequencing of all chromosomes of an organism. As an analogy with this, in 1994 the term "proteomics" was coined to refer to the complement of proteins expressed by a cell / tissue / secretion / whole organism under determined experimental conditions. This initiative has been considered the official birth of proteomics and peptidomics.

Cite: Palma, M. S. Proteomics: The Science of Functional and Structural Characterization of Proteins. Braz. J. Anal. Chem., 2020, 7 (29), pp 1-2. doi: http://dx.doi.org/10.30744/brjac.2179-3425.editorial.mspalma 
The possibility of the identification of several proteins in a single analytical round created an atmosphere of excitement surrounding proteomics, which stimulated scientists, algorithm developers and manufacturers of analytical instruments to face the challenges for the development of novel mass spectrometers, software for data treatment and the interpretation of amino acid sequencing, and HPLC systems dedicated to proteomics analysis. Thus, proteomics became a very attractive multidisciplinary area of investigation in which analytical proteomics played a pivotal role. The Brazilian Journal of Analytical Chemistry (BrJAC) may become an interesting academic forum for discussions about the development of proteomics, especially for those aspects related to the translational applications of analytical chemistry.

The Brazilian proteomics community is very active and productive, investigating a wide variety of subjects and reflecting the large biodiversity of Brazilian nature as well as themes related to agriculture and human health. To celebrate the opportunity presented by BrJAC, some contributions from leading Brazilian proteomics laboratories have been selected for this special issue. The issue presents an interview with Professor Gilberto Barbosa Domont (UFRJ) - a well-respected protein chemist based abroad and one of the pioneers of proteomics in Brazil. Professor Domont discusses aspects of his scientific career, talks about scientific politics in Brazil, and reveals his inspiration to become such an active and productive scientist. The section "Point of View" was written by Professor Marcelo Valle de Sousa (UnB) - also one of the pioneers of this field in Brazil. He presents some reflections about the development of proteomic analysis without the use of mass spectrometry. The "Letter" was written by Professor Daniel Martins de Souza (UNICAMP) - a brilliant young researcher in our community - in which he focuses on the understanding of schizophrenia by the use of MS-based proteomics. The present issue also presents two articles illustrating the state of the art of proteomics in Brazil: one is a contribution to analytical proteomics made by the study of human intestinal mucus and describes the interesting set-up conditions for the analysis of a very difficult biological matrix; the other contribution is an article describing the use of MALDI/MS and MS/MS to perform in-situ proteomic analysis accompanied by the generation of molecular images of marker proteins in slide sections of glioblastoma multiforme from the human brain for diagnostic purposes, and comparing this to the classical approach of immunohistochemistry. This issue also presents an interesting revision of the use of proteomic bioinformatics tools as an opportunity to access Systems Biology from proteomic data. We hope you enjoy reading the material in this special issue of BrJAC.

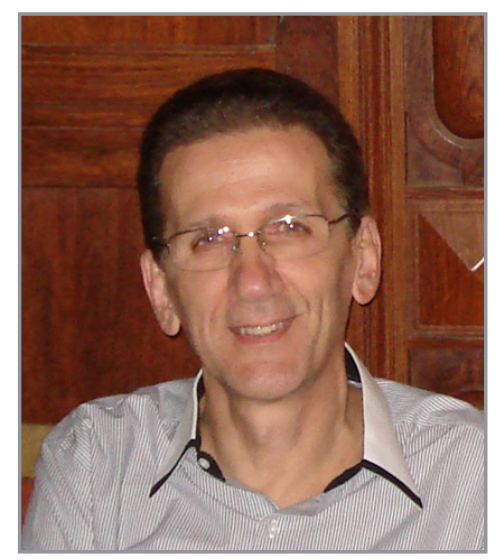

Mario Sergio Palma is a Full Professor of Proteomics at the Dept. of Basic and Applied Biology, Institute of Biosciences of UNESP Rio Claro. He got his PhD in Biochemistry at the Faculty of Medicine of São Paulo University, in Ribeirão Preto, SP, Brazil. He had internship at the Suntory Institute of Bioorganic Chemistry, in Osaka, Japan. He is a specialist in mass spectrometry of biological macromolecules and has experience in the area of Biochemistry of Macromolecules, Protein and Peptide Chemistry, as well as Chemistry of Natural Products of animal origin. He has worked in the study of the structural and functional characterization of venomous arthropod toxins, in the metabolomic and proteomic analysis of glandular secretions of insects, in the study of the structural dynamics of proteins and peptides with spectroscopic techniques. Currently, he is a member of the Core Manager of the National Institute of Science and Technology in Immunology (INCT iii) and of the Board of Directors of the Brazilian Society of Mass Spectrometry. He is currently Editor of Protein and Peptide Chemistry for the journal AMINO ACIDS (Vienna); Editor Reviewer of Frontiers in Plant Science - Section of Metabolism and Chemodiversity; member of the editorial board of the journals: Scientific Report, Peptides, Amino Acids, Toxins Reviews and Open Journal of Spectroscopy, and Toxins Reviews. He supervised 17 master's dissertations, 19 doctoral theses, 16 postdoctoral internships, and has about 300 published works. 